\title{
Kernos
}

Revue internationale et pluridisciplinaire de religion grecque antique

$14 \mid 2001$

Varia

\section{Réflexions sur les tentations du Christ au désert comparées aux genres de vie distingués par les philosophes de l'Antiquité}

Jacques Follon

\section{(2) OpenEdition}

\section{Journals}

Édition électronique

URL : http://journals.openedition.org/kernos/776

DOI : $10.4000 /$ kernos.776

ISSN : 2034-7871

Éditeur

Centre international d'étude de la religion grecque antique

Édition imprimée

Date de publication : 1 janvier 2001

Pagination : 133-146

ISSN : 0776-3824

\section{Référence électronique}

Jacques Follon, «Réflexions sur les tentations du Christ au désert comparées aux genres de vie

distingués par les philosophes de l'Antiquité », Kernos [En ligne], 14 | 2001, mis en ligne le 14 avril 2011 , consulté le 01 mai 2019. URL : http://journals.openedition.org/kernos/776 ; DOI : 10.4000/kernos.776 


\section{Réflexions sur les tentations du Christ au désert comparées aux genres de vie distingués par les phillosophes de l'Antiquité}

Comme l'indique son titre, cet article n'a d'autre prétention que de réfléchir sur un certain parallélisme que nous avons cru apercevoir entre les trois tentations du Christ au désert, telles que saint Luc les présente dans son Évangile, et le thème philosophique des trois genres de vie dans l'Antiquité classique.

Cette réflexion prend son départ dans l'observation suivante : lorsqu'on compare le récit des tentations du Christ au désert dans les deux Évangiles qui les racontent en détail (à savoir ceux de Matthieu et de Luc), on constate deux différences qui ne sont peut-être pas si minimes qu'il pourrait sembler de prime abord. Premièrement, il y a une différence dans l'ordre et la gradation des trois tentations. Chez Matthieu, en effet, on trouve d'abord la tentation de transformer les pierres en pains, puis celle de se jeter du haut du Temple pour provoquer l'intervention des anges de Dieu, et enfin celle de recevoir des mains du diable tous les royaumes de la terre avec leur gloire. Chez Luc, les deux dernières tentations sont interverties : la vision des royaumes terrestres vient en deuxième lieu et le diable réserve la tentation de se jeter du haut du Temple pour la fin. Or la plupart des commentateurs autorisés pensent que l'ordre primitif, tel qu'il aurait existé dans la fameuse source commune $Q$, est celui de Matthieu ${ }^{1}$. En effet, le déplacement du désert au Temple de Jérusalem, puis de ce dernier vers une haute montagne, représente une montée pour ainsi dire naturelle, puisqu'en allant d'un point à l'autre, on passe d'un lieu proche de la mer Morte (qui est à quelque $390 \mathrm{~m}$ en dessous du niveau de la mer), au mont Sion (qui s'élève à quelque $800 \mathrm{~m}$ au-dessus de la mer), puis de là vers une haute montagne (qui pourrait être le mont Hermon, un des lieux possibles de la Transfiguration, à 2814 mètres d'altitude). D'autre part, les passages du Deutéronome que Jésus cite pour repousser Satan sont chez Matthieu dans l'ordre exactement inverse de leur

1 A. Plummer, Critical and Exegetical Commentary on the Gospel According to S. Luke, Édimbourg, 1910 , p. 110 , a suggéré que l'ordre de Luc pourrait être "l'ordre chronologique », mais, comme le remarque J.A. FITZMYER, The Gospel According to Luke $(I-I X)$, New York, 1981, p. 507, il n'y a rien, dans le récit lucanien, qui fasse penser à une succession temporelle des tentations. 
apparition dans l'Ancien Testament ${ }^{2}$. Enfin, chez Matthieu encore, dans les deux premières tentations, le diable défie le Christ en lui disant explicitement : "Si tu es Fils de Dieu », ce qui fait penser à une paire originelle de tentations, à laquelle une troisième tentation aurait été ajoutée par la suite. On peut donc penser que Luc a délibérément modifié l'ordre primitif des tentations pour une raison bien précise. La deuxième différence entre Matthieu et Luc est que ce dernier ajoute à la fin de son récit une phrase qui ne se trouve pas chez Matthieu : "Ayant ainsi épuisé toute tentation, le diable s'éloigna de lui jusqu'au moment favorable $»^{3}$.

Diverses explications ont été données de ces deux différences entre les deux récits évangéliques de la tentation au désert.

Tout d'abord, à la question de savoir pourquoi Luc a modifié l'ordre originel des tentations, les commentateurs ont donné des réponses assez différentes. Selon K.H. Rengstorf, Luc (ou sa source propre) aurait opéré cette modification de manière à ce que le nouvel ordre soit le reflet inversé des trois premières demandes de la version lucanienne du Pater: «Père, que ton Nom soit sanctifié, que ton Règne vienne, donne-nous chaque jour notre pain quotidien ${ }^{4}$. Pour H. Swanston, l'ordre de Luc serait à mettre en rapport avec le Psaume $106^{5}$. Mais ces deux explications pèchent sans doute par un excès de subtilité. En fait, la majorité des commentateurs expliquent la différence d'ordre des tentations chez les deux évangélistes plutôt par leur conception différente du point culminant de l'Évangile. C'est ainsi que Matthieu aurait situé sur une haute montagne la tentation liée à la vision des royaumes du monde, soit en raison de l'importance du thème de la montagne dans son propre Évangile, soit à cause du caractère décisif de la réponse donnée par le Christ à cette tentation : «Tu adoreras le Seigneur ton Dieu, et à lui seul tu rendras un culte ». De son côté, Luc aurait inversé l'ordre des deux dernières tentations en raison de l'importance qu'il accorde à Jérusalem, la Ville Sainte, dans l'histoire du salut : « Jérusalem est la ville où Jésus doit accomplir son départ, où il doit souffrir sa passion. Il convenait que ce fût là que les tentations s'achèvent et que le diable se retirât de lui ${ }^{6}$. En outre, remarque un des meilleurs commentateurs actuels, pour Luc, « la question, certes délicate, mais avant-dernière du pouvoir politique ne mérite pas d'occuper la dernière place, la plus importante. Selon la gradation de Luc, c'est tenter Dieu qui représente la plus grave tentation ${ }^{7}$. En ce qui concerne

\footnotetext{
2 Mt 4, 4 correspond à $D t 8,3 ; M t 4,7$ à $D t 6,16$; et $M t 4,10$ à $D t 6,13$.

3 Lc 4, 13. N.B. : sauf indication contraire, toutes nos traductions des passages de l'Écriture sont empruntées à la Bible de Jérusalem.

4 K.H. Rengstorf, Das Evangelium nach Lucas, Göttingen, 1978, p. 63.

5 H. Swanston, "The Lukan Temptation Narrative", Joumal of Theological Studies 17 (1966), p. 71.

6 J. Dupont, Les tentations de Jésus au désert, Bruges, 1968, p. 70.

7 F. Bovon, L'Évangile selon saint Luc (1,1 - 9,50], Genève, 1991, p. 189.
} 
l'affirmation, spécifique à Luc, qu'en ayant soumis Jésus aux trois tentations que l'on sait, le diable avait ainsi épuisé toutes les formes de la tentation, on a suggéré qu'il s'agirait là d'un écho de l'idée juive selon laquelle « seule la triple mention d'une action, le triple accomplissement d'un événement en font quelque chose d'achevé et de définitif ${ }^{8}$. Cependant, lorsqu'on connaît quelque peu la philosophie antique, on ne peut qu'être frappé par le fait que les principaux genres de vie, ainsi que les fonctions de l'âme et leurs déviations correspondantes, y étaient précisément aussi au nombre de trois. Est-ce un hasard ? C'est possible - beaucoup diront même que c'est certain -, mais il faut quand même se rappeler que Luc était imprégné de culture grecque et qu'il avait indéniablement une certaine connaissance de la philosophie païenne ${ }^{9}$. Aussi la question, ne disons pas encore ou pas nécessairement d'une influence, mais au moins d'un rapprochement ou d'un parallélisme entre ce passage de Luc et les textes philosophiques traitant de ces genres de vie mérite-t-elle, à nos yeux, d'être posée.

D'après une tradition, dont on trouve un écho dans les Tusculanes de Cicéron et chez Diogène Laërce, mais qui remonte à Héraclide du Pont, philosophe platonicien du $\mathrm{IV}^{\mathrm{e}}$ siècle av. J.-C., le thème des genres de vie ${ }^{10}$ aurait été introduit dans la philosophie grecque par Pythagore. En effet, alors qu'il discutait avec un certain Léon, «tyran » de Phlionte (ou de Sicyone), Pythagore aurait expliqué qu'il était un «philosophe » ( $\phi\llcorner\lambda o ́ \sigma o \phi o s)$. Et comme Léon ne connaissait pas ce mot nouveau et lui demandait ce qui différenciait les philosophes et le reste des hommes, Pythagore lui aurait répondu :

que la vie humaine lui paraissait semblable à cette assemblée où étaient organisés les jeux que fréquentait la Grèce entière; là, les uns ayant exercé leur corps venaient chercher la gloire et l'illustration d'une couronne; d'autres, venus pour acheter ou pour vendre, y étaient conduits par l'appât du gain; mais il y avait une sorte de visiteurs (et même particulièrement distingués) qui ne cherchaient ni les applaudissements ni le gain, mais qui venaient pour voir et qui examinaient avec grand soin ce qui avait lieu et comment les choses se passaient. De même que tous ceux-là sont partis de leur ville pour la célébration des jeux, de même les hommes venus à cette vie

\section{G. Delling, cité par F. Bovon, ibid., p. 189.}

9 Ainsi le discours de S. Paul devant l'Aréopage d'Athènes ( $A C$ 17, 22-31) comporte d'assez nombreuses allusions aux doctrines des philosophes païens. Luc les explique par le fait que, durant son escale à Athènes, Paul, dont il était le compagnon, "s'entretenait [...] sur l'agora, tous les jours, avec les passants " et que là " il y avait même des philosophes épicuriens et stoïciens qui l'abordaient" (Ac 17, 17-18). Voir, à ce sujet, J. Follon, "Suiure la Divinité ". Introduction à l'esprit de la philosophie ancienne, Louvain/Paris, 1997, p. $213 \mathrm{sq}$.

10 Sur ce thème, l'étude la plus complète reste celle de R. Joly, Le thème philosophique des genres de vie dans l'Antiquité classique, Bruxelles, 1956 (Mémoires de la Classe des Lettres et des Sciences morales et politiques de l'Académie royale de Belgique, $51,3)$. 
humaine en quittant une autre vie et une autre nature, sont les uns esclaves de la gloire, les autres, de l'argent; mais il en est de bien rares qui, comptant pour rien tout le reste, observent avec soin la nature; ce sont eux qu'on appelle amis de la sagesse, c'est-à-dire philosophes; et de même que, à l'assemblée des jeux, l'attitude la plus digne d'un homme libre est de regarder, sans rien gagner, de même dans la vie, la contemplation et la connaissance des choses l'emportent de beaucoup sur tous les autres travaux ${ }^{11}$.

Bref, si l'on en croit cette histoire, Pythagore aurait bien distingué trois sortes d'hommes en fonction du but poursuivi par eux dans la vie : il y a d'abord ceux qui vivent pour faire des affaires, gagner de l'argent et jouir des biens de ce monde, ensuite ceux qui recherchent la gloire et les honneurs (les uns comme les autres ayant une nature d'esclave), enfin les philosophes, qui n'ont d'autre but dans la vie que de contempler la nature et de connaître la vérité des choses et qui, de ce fait, sont les seuls hommes vraiment libres ${ }^{12}$.

Après Pythagore, Platon et Aristote devaient reprendre et approfondir ce thème des trois genres de vie. Ainsi, dans la République, Socrate montre à son tour que les hommes vivent pour l'une ou l'autre des trois choses suivantes : la richesse, les honneurs ou la sagesse. On peut donc, dit-il aussi, diviser l'humanité en trois genres d'individus ${ }^{13}$. Mais l'originalité de Platon est de mettre ces trois genres d'hommes en rapport avec les trois parties de l'âme et les trois classes sociales de la cité : aux amoureux des richesses correspondent l'âme concupiscible (ou principe du désir) et la classe des producteurs; aux amoureux de la gloire et des honneurs, l'âme irascible (ou principe du

11 Cicḱron, Tusc. V, III, 9 (trad. É. Bréhier, in Les Stoïciens [Bibliothèque de la Pléiade], p. 364), Cf. Jamblique, Vie pythagorique, $₫ 58$.

12 Certains critiques, comme W. Burkert ("Platon oder Pythagoras? Zum Ursprung des Wortes ,Philosophie'", Hermes 88 [1960], p. 159-177) ou P. Hadot (Qu'est-ce que la philosophie antique?, Paris, 1995 , p. 35, n. 1), pensent que l'anecdote n'a pu naître que dans l'Académie de Platon, pour illustrer l'idéal platonicien de la vie contemplative, si bien qu'elle aurait été forgée de toutes pièces par Héraclide, qui l'aurait appliquée rétrospectivement à Pythagore. Mais, comme le dit bien C.J. DE Vogel ("Some Reflections on the Term $\phi i \lambda o \sigma o \phi i a "$, in Philosopbia I: Studies in Greek Philosophy, Assen, 1970, p. 78) : «Nous avons de bonnes raisons d'admettre que Pythagore lui-même s'est donné le

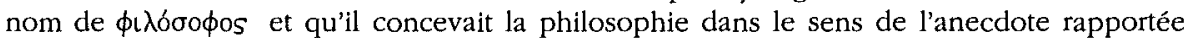
plus haut : c'est que, pour lui et pour ses disciples, la philosophie était bien une $\theta \in \omega p l a$ et que cela impliquait une certaine attitude personnelle devant la vie, à savoir une attitude de liberté intérieure et de distanciation par rapport aux choses convoitées par la plupart des hommes : l'argent et les biens matériels, le confort et le plaisir des sens, et même les honneurs et la considération d'autrui. " Pour une critique plus approfondie de la position de Burkert, voir C.J. De Vogen, Pythagoras and Early Pythagoreanism, Assen, 1966, p. 96-102, et R. Joly, "Platon ou Pythagore? Héraclide Pontique, fr. 87-89 Wehrli", in Glane de philosopbique antique, Bruxelles, 1994, p. 15-31.

$13 \mathrm{Cf}$. Républ. IX, 581c : «C'est pour cela que nous disions qu'il y a trois principales classes d'hommes: le philosophe, l'ambitieux, l'intéressé [litt. l'ami de la sagesse

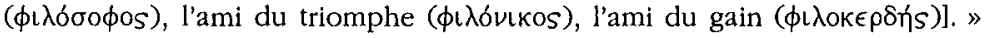


courage) et la classe des guerriers; et aux amoureux de la sagesse (c'est-à-dire aux philosophes), l'âme rationnelle (ou principe de la pensée) ${ }^{14}$. Nous pouvons donc penser que Platon considérait cette énumération des trois genres de vie comme exhaustive. Cependant, continue Socrate, si l'on demande, à chacun de ces trois types d' " amoureux » (des richesses, des honneurs ou de la sagesse), quelle sorte de vie donne le plus de plaisir, chacun répondra évidemment que c'est la sienne. Ainsi l'homme d'affaires trouvera certainement que le plaisir de gagner de l'argent est plus substantiel que le plaisir procuré par les honneurs politiques ou par la découverte scientifique, tandis que celui qui aime les honneurs tiendra pour vulgaire le plaisir d'amasser une fortune et pour une frivolité celui d'accumuler les connaissances. Mais le philosophe, lui, trouvera évidemment son plus grand plaisir dans la saisie de la vérité et il ne verra la satisfaction des besoins naturels et les obligations de la vie publique (y compris le service militaire) que comme de regrettables nécessités. Qui a raison? Pour le savoir, il faut s'en remettre au seul critère qui permette de juger correctement en cette matière, à savoir l'expérience. Or, de ce point de vue, on doit admettre que le philosophe, comme n'importe qui d'autre, a goûté dès l'enfance au plaisir du gain, et que, si son enseignement a connu un certain succès, il aura aussi goûté au plaisir des honneurs, car le sage n'est pas moins honoré que le soldat courageux et le riche. C'est pourquoi son expérience de la vie comprend celle de ces deux autres types d'hommes, en même temps qu'elle la dépasse, tandis qu'eux ne savent rien du plaisir procuré par la découverte et la contemplation des vraies réalités. Aussi le philosophe est-il le seul qui soit à même de comparer et d'évaluer les différentes sortes de plaisirs. Au demeurant, le plaisir goûté dans la vie philosophique est supérieur aux plaisirs éprouvés dans les autres sortes de vie ${ }^{15}$. Car la majorité des plaisirs corporels ne sont jamais que le remplissage d'un vide douloureux ou le soulagement d'une gêne. Ce ne sont donc pas des plaisirs purs ou vrais (c'està-dire non mélangés de douleur), et celui qui les regarde comme des plaisirs positifs est semblable à quelqu'un qui se trouve à la moitié d'une montée et qui, ne pouvant voir plus haut, s'imagine être arrivé au sommet, ou encore il ressemble à quelqu'un qui, n'ayant jamais vu du blanc, ferait du gris le contraire du noir. Or, de même que la faim et la soif sont des sortes de vide dans le corps, de même l'ignorance et la déraison sont aussi des sortes de vide, mais dans l'âme. Cependant, étant donné que les choses qui remplissent l'esprit sont, comme l'esprit lui-même, immuables et éternelles, elles sont, par là même, plus réelles que les choses matérielles, qui sont toujours changeantes et éphémères. Dès lors, l'esprit, qui se remplit « de choses plus réelles et qui est lui-même plus réel », sera plus réellement rempli que le corps, qui « se remplit de choses moins réelles et qui est lui-

14 Républ. IV, 436ab; IX, 580d-581e.

15 Ibid., $583 \mathrm{~b}-585 \mathrm{e}$. 
même moins réel ${ }^{16}$. D'où il faut conclure qu'étant ainsi plus réellement rempli de choses plus réelles, l'esprit, lui-même plus réel que le corps, jouit par là « plus réellement et plus véritablement du vrai plaisir ».

Pour ce qui est d'Aristote, on sait qu'il s'est également attaché à montrer la supériorité de la vie philosophique sur les autres genres de vie dans le premier livre de l'Éthique à Nicomaque. Ainsi, lui aussi déclare que les principaux genres de vie sont au nombre de trois. Il y a d'abord la vie de plaisir, qui est au fond une vie bestiale et qui n'est choisie que par les gens les plus grossiers et dotés d'une mentalité d'esclave (même s'ils appartiennent à la classe dirigeante). Ensuite, il y a la vie de l'homme politique, qui vaut déjà mieux, puisque ce qu'on y recherche est l'honneur, qui est une fin plus noble que le plaisir et les biens matériels. Il n'empêche que l'honneur ne saurait davantage constituer le bien suprême, car " il dépend plutôt de ceux qui honorent que de celui qui est honoré ", alors que "nous savons d'instinct que le bien est quelque chose de personnel à chacun et qu'on peut difficilement nous ravir ». Aristote évoque encore la vie de l'homme d'affaires, mais il n'en fait pas une quatrième sorte de vie, puisqu'elle est, comme la vie de plaisir, une vie de contrainte et que, pas plus que le plaisir ou les honneurs, la richesse ne saurait être le bien suprême, étant « seulement une chose utile, un moyen en vue d'une autre chose ", à savoir précisément le plaisir ou les honneurs. Reste donc le troisième genre de vie, qui est axé sur la contemplation $^{17}$. Mais, pour être sûr que c'est bien celle-ci qui rend l'homme vraiment heureux et constitue donc pour lui le bien suprême, il est nécessaire, dit le Stagirite, d'explorer plus avant la nature du bonheur humain. Pour ce faire, il faut partir de la fonction qui est propre à l'homme en tant qu'bomme. Celleci ne peut être que l'activité spécifique qui le distingue des autres espèces d'animaux, à savoir l'activité de l'âme rationnelle ${ }^{18}$. Or, si le bien, pour tout individu, consiste à accomplir bien et avec succès la fonction qui lui est propre (par exemple, « la fonction du cithariste est de jouer de la cithare, et celle du bon cithariste d'en bien jouer »), alors le bien de l'homme en tant qu'homme ne sera pas autre chose que le bon accomplissement de l'activité de son âme rationnelle. Mais le bon accomplissement d'une fonction ou d'une activité repose sur la possession d'une vertu. Car la vertu d'une chose, c'est justement la disposition qui non seulement met cette chose en bon état, mais aussi lui permet de bien accomplir sa fonction (par exemple, c'est par la vertu de l'œil que notre vision est bonne, tout comme c'est par la vertu du cheval que celui-ci est bon pour la course, porte bien son cavalier et fait face à l'ennemi). Par conséquent, pour l'homme, le souverain bien, qui se confond avec le bonheur, consistera dans l'activité de l'âme rationnelle conforme à la vertu propre de celle-ci (la oo $\phi(\alpha)$, c'est-à-dire dans l'activité de la partie de nous-mêmes qui est la plus baute (car c'est elle qui $1^{\circ}$ nous commande et

16 Ibid., 585d.

17 Eth. Nic. I, 2-3.

18 Ibid. I, 6, 1097b 24 - 1098a 5. 
nous dirige, $2^{\circ}$ possède la connaissance de ce qui est noble et divin, $3^{\circ}$ est la partie de notre être la plus divine). Et cette activité de l'âme rationnelle sera la contemplation des substances divines, surtout de la plus élevée d'entre elles: le Premier Moteur ${ }^{19}$, principe suprême auquel « sont suspendus le Ciel et la nature $»^{20}$. Enfin, il faut souligner que, pour Aristote, cette vie contemplative (ou «vie selon l'intellect») était ce qui rapprochait le plus l'homme de la condition divine :

car ce n'est pas en tant qu'homme qu'on vivra de cette façon, mais en tant que quelque élément divin est présent en nous. [...] Si donc l'intellect est quelque chose de divin par comparaison avec l'homme, la vie selon l'intellect est également divine comparée à la vie humaine. Il ne faut donc pas écouter ceux qui conseillent à l'homme, parce qu'il est homme, de borner sa pensée aux choses humaines, et mortel, aux choses mortelles, mais l'homme doit, dans la mesure du possible, s'immortaliser, et tout faire pour vivre selon la partie la plus noble qui est en lui ${ }^{21}$.

Après avoir vu brièvement comment le thème des genres de vies avait été traité par des philosophes païens, nous pouvons revenir au passage de Luc dont nous sommes partis. La première tentation du Christ peut être rapprochée, sans trop de difficultés, de la vie du jouisseur ou de l'homme d'affaires dont parlait Aristote et qui donnent la première place dans la hiérarchie des valeurs aux biens matériels, alors que ceux-ci ne sont que des biens inférieurs ou subordonnés, qui ne sauraient prétendre au titre de biens suprêmes et de fins ultimes dans la vie humaine. Le Christ, en effet, répond clairement au diable, qui l'invite à transformer des pierres en pains, que « l'homme ne vit pas seulement de pain, mais de toute parole qui sort de la bouche de Dieu ${ }^{22}$, ce qu'il est facile de paraphraser en disant que l'homme n'a pas seulement besoin de nourritures terrestres, mais aussi, et plus fondamentalement encore, de nourritures spirituelles, en l'occurrence de la sagesse divine contenue dans la Révélation. Notons que, pas plus que Platon ou Aristote, le Christ ne condamne la recherche des biens matériels (le « pain ») comme un mal en soi, mais que pour lui, comme pour eux, cette recherche devient une chose mauvaise lorsqu'elle aboutit à exclure toute quête' d'un bien supérieur.

La chose n'est pas moins claire pour la deuxième tentation: le diable

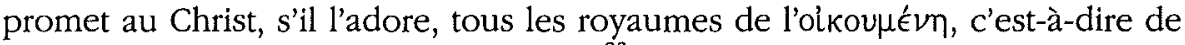
la terre habitée, avec leur gloire $(\delta \delta \xi \alpha)^{23}$. Pour celui qui est un tant soit peu familier de la philosophie grecque, il est difficile de ne pas voir ici un parallèle

Ibid. X, 7, 1177a 12-21. Cf. Métaph. VI, 1, 1026a 10 sq.; XII, 8.

Métaph. XII, 7, 1072b 14.

21 Ëth. Nic. X, 7, 1177b 27 sq.

22 Mt 4, 4, qui est une citation de Dt 8, 3. La citation donnée par $L c 4,4$ ne comprend que la première partie de la phrase, mais elle a évidemment le même sens.

23 Lc 4, 6; cf. Mt 4, 8-9. 
avec la description de la vie de l'homme politique assoiffé d'honneurs, de triomphes et de puissance.

En revanche, pour ce qui est de la troisième tentation, il faut reconnaître qu'un parallèle avec une éventuelle déviation de la vie de sagesse est beaucoup plus difficile à établir. Aujourd'hui, en effet, cette tentation est communément interprétée de la manière suivante : le diable y met le Christ au défi de prouver qu'il est bien le Fils de Dieu en faisant un miracle spectaculaire, consistant à se jeter du haut du Temple et à demander à son Père l'assistance de ses anges, «car il est écrit : Il donnera pour toi des ordres à ses anges, afin qu'ils te gardent. Et encore : Ils te porteront dans leurs mains, de peur que tu ne beurtes du pied quelque pierre ». À quoi le Christ répond : «Il est dit : Tu ne tenteras pas le Seigneur, ton Dieu » ${ }^{24}$. Or, si le Christ repousse sans hésiter cette troisième tentation, c'est parce qu'il sait que l'accepter reviendrait à tenter Dieu. Mais mettre Dieu à l'épreuve, c'est lui refuser sa confiance. C'est, par exemple, s'exposer de manière présomptueuse à un danger inutile, comptant sans raison sur une aide extraordinaire de Dieu. Tenter Dieu, c'est aussi lui réclamer des signes pour pallier l'incrédulité ou l'arrogance humaine ${ }^{25}$.

De prime abord, il semble bien qu'il n'y ait aucun rapport entre l'épreuve à laquelle le diable soumet ainsi Jésus et la vie de sagesse. Toutefois, en parcourant le contexte plus large où le récit de la troisième tentation s'inscrit, à savoir l'extrait du psaume 91 qui est cité par le diable, il n'est peut-être pas impossible de déceler un tel rapport.

Voyons tout d'abord de plus près la citation du psaume 91 :

Si tu es fils de Dieu, dit le diable à Jésus, jette-toi d'ici [le pinacle du Temple] en bas, car il est écrit [dans le psaume 91] : "Il donnera pour toi des ordres à ses anges, afin qu'ils te gardent». Et encore : «Sur leurs mains, ils te porteront, de peur que tu ne heurtes du pied quelque pierre ».

Il faut savoir que ce psaume est un poème didactique sur la protection complète que Dieu donne à celui qui s'abrite en lui avec une entière confiance :

24 Lc 4, 11-12.

25 La Bible. L'Évangile selon saint Matthieu. Textes français et latin. Commentaires réalisés par la Faculté de Théologie de l'Université de Navarre, Paris, éd. du Laurier, 1994, p. 69. C'était déjà l'interprétation de plusieurs Pères de l'Église, comme S. Irénée de Lyon (Adv. Haer. V, 21, 2). Mais certains Pères donnaient une interprétation différente, entendant la tentation du diable comme une invitation adressée au Christ pour qu'il fasse étalage de sa toute-puissance divine. Ainsi, S. Hilaire de Poitiers (In Matthaeum III, 4) : «Brisant les efforts et les tentatives du diable, il [Jésus] s'affirme comme Dieu et Seigneur, enseignant que l'arrogance ne doit pas avoir de place chez les croyants. " De même, S. Ambroise (In Lucam, IV, 27) fait suivre la réponse de Jésus par ces mots : "Par où l'on connaît que le Christ est le Seigneur Dieu, et que le Père et le Fils n'ont qu'une même puissance. " 


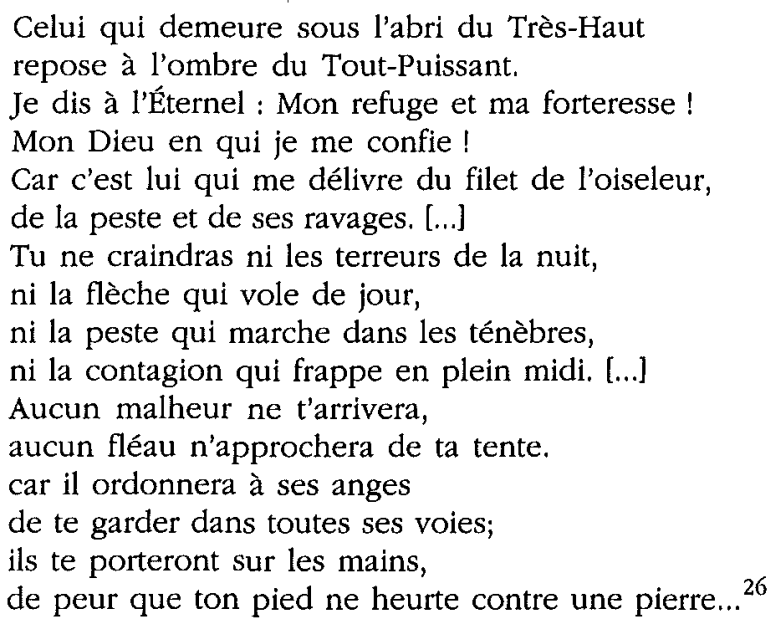

Or celui qui s'abrite ainsi en Dieu est clairement présenté, dans le livre des Proverbes, comme un juste : « le nom de l'Éternel est une tour forte; le juste s'y réfugie, et se trouve en sûreté ${ }^{27}$. Cependant, le contexte de cette phrase des Proverbes montre bien que le juste en question est identiquement quelqu'un qui, par son comportement, s'oppose à l'insensé, lequel vit à l'écart de la loi de Dieu, provoque des querelles, se livre à la calomnie, se complaît dans la paresse, se fie à la richesse, et finalement entraîne sa propre perte par tous ces comportements mauvais. Puisqu'il s'oppose ainsi à l'insensé, le juste apparât donc aussi comme un sage, dont le cœur intelligent « acquiert la science » et dont l'oreille « recherche le savoir ${ }^{28}$. Autrement dit, pour l'auteur des Proverbes, la justice et la sagesse ne font qu'un, parce qu'elles consistent essentiellement dans la même chose : se conformer parfaitement à la volonté de Dieu, observer scrupuleusement ses lois, suivre ses voies en toutes choses ${ }^{29}$. Comme le dit alors le psaume 91, c'est bien pour celui qui fait montre d'une telle sagesse que le Seigneur mobilisera ses anges, afin qu'ils le gardent dans toutes ses voies et le portent sur leurs mains, de peur que son pied ne heurte une pierre. On pourrait donc peut-être relier la troisième tentation du Christ à la sagesse de celui qui suit fidèlement les voies du Seigneur et qui, pour cette raison, peut compter sans réserve sur sa protection. Et peut-être aussi pourrait-on alors paraphraser les paroles que le

26 Trad. Segond revue (1975).

$\operatorname{Pr} 18,10$ (tr. Segond).

28

$\operatorname{Pr} 18,15$. Voir aussi l'hymne à la sagesse au début du Siracide.

29 Notons qu'en un sens, les stoïciens (que Luc connaissait bien) pensaient exactement la même chose. $C f$. DIOGÈne LAËrCE, VII, 119 : Les sages « sont purs. Ils repoussent en effet toutes les fautes contre les dieux. Les dieux mettent en eux leur complaisance, car ils sont saints et justes envers le divin " (trad. $\mathrm{R}$ Goulet et M. Patillon). Voir aussi les autres références citées infra, n. 39-40. 
diable adresse à Jésus de la manière suivante : «Si tu es fils de Dieu, c'est-àdire : si tu es un juste ou, ce qui revient au même, un sage (parce que tu te conformes entièrement à sa volonté), alors demande-lui sa protection spéciale et tu deviendras son favori, au service duquel il n'hésitera pas à mettre ses anges ».

En quoi consiste exactement le péché qui correspond à cette tentation ? On aura compris qu'il consiste évidemment à considérer que la protection de Dieu nous est due lorsque nous sommes des « justes » ou des « sages », c'està-dire, encore une fois : lorsque nous nous conformons entièrement à sa volonté et que nous obéissons à tous ses commandements, comme si cette obéissance nous donnait en quelque sorte des droits sur lui. C'est le péché d'orgueil typique des pharisiens, que le Christ dénoncera implacablement, notamment dans la parabole du pharisien et du publicain venus prier au Temple $^{30}$. C'est aussi le péché de l'ambition, qui ne concerne plus, cette fois, la gloire et la domination terrestres, mais bien les valeurs spirituelles, car il consiste à prétendre détenir la connaissance suprême ou divine, qui ferait que nous serions « comme Dieu » et que nous mériterions ainsi d'être servi par ses anges...

Or, de ce point de vue, il faut reconnaitre qu'il existait, dans la philosophie païenne, une solide tradition faisant de la sagesse du philosophe une qualité véritablement divine. Ainsi Pythagore aurait inventé le mot $\phi$ idoбoфía pour signifier qu'au sens strict du terme, « nul n'est sage, si ce n'est la divinité ${ }^{31}$, mais cela n'aurait pas empêché ses disciples de le considérer comme un dieu (ou, à tout le moins, un demi-dieu) en raison, précisément, de son immense sagesse ${ }^{32}$. De même, pour Empédocle, les âmes des sages étaient appelées à devenir des dieux ${ }^{33}$. Et l'on sait que Socrate considérait que sa vraie patrie était le $\mathrm{ciel}^{34}$. Par ailleurs, vers la fin de sa vie, Platon avait fini par assimiler la véritable piété à la sagesse même, laquelle, d'ailleurs, consistait essentiellement pour lui dans la connaissance de l'astronomie et des mathématiques ${ }^{35}$. Enfin, nous avons vu, ici même, que pour Aristote, la vie du sage était, aussi au sens quasi littéral du terme, une vie divine. Ajoutons que les épicuriens et les stoïciens pensaient pratiquement la même chose, certes pour des raisons quelque peu différentes, mais qui n'en aboutissaient pas moins à faire du sage un « dieu parmi les hommes ${ }^{36}$, selon l'expression d'Épicure lui-même. Ce dernier, en effet, estimait que le sage est tel, parce

30 LC, 18, 9-14.

31 DIOGÈnE LAËRCE, I, 12.

32 Cf. DIOGÈne LAËrCE, VIII, 11; ÉlIEN, Histoires variées II, 26; IV, 17; Jamblique, Vie pythagorique, $\$ 30-31,140$.

33 Empédocle, fr, 146 (= Clément d'Alexandrie, Stromates IV, 150).

34 Voir, entre autres, le Phédon (passim), ainsi que le Théétète (176a-b).

35 Épinomis, 989a sq.

36 ÉPICURe, Lettre à Ménécée, 135. 
que, grâce à la philosophie, il vit dans une absence de troubles et une parfaite sérénité qui sont des biens impérissables, et que « celui qui vit au milieu de biens impérissables ne ressemble en rien à un être mortel ${ }^{37}$. Quant aux stoïciens, ils soutenaient que le sage est l'homme qui vit en suivant la nature, non seulement la sienne propre, mais aussi celle de l'univers tout entier, sans rien faire « de ce que défend la loi commune, c'est-à-dire la droite raison qui circule à travers toute chose et qui est identique à Zeus, le chef du gouvernement de l'univers ${ }^{38}$. Selon eux, ce sage devait regarder le même Zeus, identifié au Dieu suprême, comme le Père de tous les êtres ${ }^{39}$ et lui demander essentiellement, dans une prière filiale : "Laisse-nous participer à cette sagesse sur laquelle tu te fondes pour gouverner toutes choses avec justice ${ }^{40}$. Et ils ajoutaient que, dans la mesure où il ne désire rien d'autre que ce qui lui arrive, le sage n'est l'esclave d'aucun manque et d'aucun besoin, de sorte qu'il vit, totalement libre, dans un état de parfaite auto-

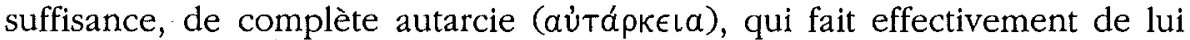
l'égal d'un dieu ! ${ }^{41}$ Or rappelons, une fois encore, que Luc devait avoir une certaine connaissance au moins des épicuriens et des stoïciens...

Cependant, pour notre propos, la question décisive semble être celle-ci : l'évocation d'un miracle aussi spectaculaire que gratuit, comme celui de se jeter dans le vide du haut du Temple sans en subir aucun dommage, était-elle susceptible d'éveiller, dans l'esprit d'un grec cultivé du premier siècle de notre ère, l'idée d'une manifestation de la « sagesse » d'un personnage possédant des « pouvoirs » spirituels hors du commun ? À notre humble avis, ce n'est pas impossible. En effet, selon plusieurs biographies antiques, Pythagore, qui incarnait pour beaucoup le sage par excellence, aurait été, comme nous venons de le voir, un dieu ou, à tout le moins, un demi-dieu, qui aurait accompli divers miracles spectaculaires manifestant son pouvoir sur les animaux et même sur la nature proprement dite. C'est ainsi qu'on disait qu'il avait réussi à convaincre une ourse de Daunia (en Italie du sud) de ne plus s'attaquer aux hommes et aux bêtes, et aussi un bouf de Tarente de s'abstenir de manger des fèves ${ }^{42}$. Bien plus, non content d'exhiber à plusieurs reprises sa fameuse cuisse en or ${ }^{43}$, il aurait été capable d'éloigner des épidémies $^{44}$, de mettre fin instantanément à des vents violents et des tempêtes de

\footnotetext{
37 Ibid.

38 Diogène LAËrce, VII, 88 (trad, Bréhier).

39 Ibid. VII, 147.

40 Cléanthe, Hymne à Zeus, 35 (trad. Bréhier)

41 Diogìne LaËrce, VII, 119; Stobée, Églogues II, 198; Plutarque, Des notions communes contre les stoïciens, 33; Des contradictions des stoïciens, 13.

42 Porphyre, Vie de Pythagore, $\$ 23-24$; Jamblique, Vie pythagorique, $\$ 60-61$.

43 Porphyre, Vie de Pythagore, $₫ 28$; Jamblique, Vie pythagorique, $\$ 92,135,140$; Diogène Lä̈rce, VIII, 11; Plutarque, Vie de Numa, 8; Élien, Histoire variée, IV, $26,17$.

44 Porphyre, Vie de Pythagore, $\$ 29 ;$ JAmblique, Vie pythagorique, $₫ 135$.
} 
grêle ${ }^{45}$, d'apaiser les flots irrités des fleuves ou de la mer ${ }^{46}$, de se faire saluer, « d'une voix grave et distincte », par le fleuve $\operatorname{Nessos}^{47}$, et même de se rendre maître de l'espace en se transportant en un instant de Métaponte à Crotone ${ }^{48}$, à Tauroménion ${ }^{49}$ ou à Sybaris $^{50}$, alors qu'il fallait plusieurs jours pour accomplir ces voyages... ${ }^{51}$ Et il n'aurait pas été le seul « sage » de cette espèce, car Jamblique, se référant sans doute à des traditions anciennes, rapporte que les philosophes Empédocle, Épiménide et Abaris, « qui avaient part aux mêmes pouvoirs, ont accompli de semblables actions en beaucoup d'endroits $»^{52}$. Enfin, on ne saurait passer sous silence la figure du philosophe néo-pythagoricien contemporain du Christ Apollonios de Tyane, dont on disait que, par la sagesse et la méditation, il était arrivé, comme Pythagore luimême, à se libérer des besoins physiques et à acquérir des dons extraordinaires, tels que la compréhension du langage des oiseaux, la connaissance de toutes les langues humaines, la vision du secret des cœurs, la divination, l'art de guérir des possédés, et même la capacité de faire les miracles les plus spectaculaires : c'est ainsi que l'on racontait qu'il avait ressuscité une jeune fille morte le jour de son mariage ${ }^{53}$ et que lui-même avait quitté le monde d'ici-bas au milieu des plus grands prodiges : arrivé dans un temple à une heure indue, il aurait été arrêté et enchaîné comme sorcier et voleur par les prêtres du lieu, mais il se serait défait sans difficulté de ses liens et, devant les prêtres ébahis, se serait élancé vers la porte du temple, laquelle se serait ouverte puis refermée toute seule, alors qu'à l'intérieur, il s'élevait vers le ciel... ${ }^{54}$ Il semble donc bien que, dans une certaine tradition philosophique, l'image du «sage » était associée à la possession de pouvoirs extraordinaires sur la nature, comprenant notamment la maîtrise de l'espace et des forces naturelles, qui joue précisément un rôle dans la troisième tentation du Christ, puisque, après une translation instantanée d'une haute montagne au Temple de Jérusalem, le Messie est invité à défier, en quelque sorte, la loi de la chute des corps...

Dans une telle perspective, cette troisième tentation recouvrirait alors un mauvais usage de la sagesse divine, comme les deux premières recouvraient

45 Ibid.

46 Ibid.

47 JaMblique, Vie pythagorique, $₫ 134$; DIOGÈne LAËrRC, VIII, 11.

48 apollonios le Paradoxographe, Mirabilia, 6.

49 Porphyre, Vie de Pythagore, $₫ 27$ et 29; Jamblique, Vie pythagorique, $\$ 134$.

50 Philostrate, Vie d'Apollonios de Tyane IV, 10 (Thourioi est mis pour Sybaris).

51 Cette maitrise de l'espace ne serait-elle pas à rapprocher de celle du Christ se transportant en un instant, avec le diable, du désert à Jérusalem et susceptible de se jeter dans le vide sans se blesser?

52 Jamblique, Vie pythagorique, $\$ 135$. Cf. Porphyre, Vie de Pythagore, $₫ 29$.

53 Philostrate, Vie d'Apollonios de Tyane IV, 45.

54 Ibid. VIII, 30. 
un mauvais usage l'une des richesses, l'autre du pouvoir politique. Certes, les philosophes intellectualistes qu'étaient Socrate et Platon auraient eu des difficultés à envisager un tel mauvais usage de la sagesse, entendue du moins dans son sens véritable, parce qu'ils étaient convaincus qu'aucun homme possédant la (vraie) sagesse ne pouvait faire le mal volontairement. Pour eux, la possession de cette sagesse, qui était à leurs yeux à la fois intellectuelle et morale, ne pouvait conduire qu'à une vie et à des actions bonnes. C'est dire qu'ils ne concevaient pas que l'on pût être à la fois sage et « malin » (au sens moral du terme). Il n'en reste pas moins que, dans la République, Platon

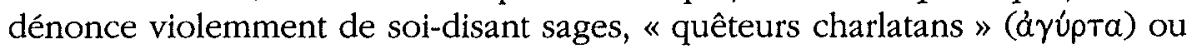
« devins » ( $\mu a ́ v T \epsilon \iota s)$, qui font croire aux riches « qu'au moyen de sacrifices et d'incantations, ils ont obtenu des dieux le pouvoir de réparer, par des jeux et des fêtes, n'importe quelle faute commise par un homme ou par ses ancêtres, et que si l'on veut faire du tort à un ennemi, il n'en coûtera pas beaucoup pour nuire au juste aussi bien qu'à l'injuste, puisqu'ils sont, disent-ils, maîtres des incantations et des formules magiques qui contraignent les dieux à servir leurs fins ${ }^{55}$. De même, dans la cité des Lois, de lourdes peines de prison sont prévues pour ceux « qui promettent de convaincre les dieux avec la pensée que, par des sacrifices, par des prières, par des incantations, ils les ensorcellent ${ }^{56}$.

Il est évidemment impossible de prouver que, dans sa présentation des tentations du Christ au désert, Luc ait été le moins du monde influencé par le thème des genres de vie cher aux philosophes grecs. Mais nous persistons à croire qu'une telle hypothèse n'est pas totalement à exclure. Car on sait qu'il écrivit son Évangile à l'intention de chrétiens de culture grecque. Or Robert Joly a pu montrer qu'après le premier siècle avant notre ère, le thème des genres de vie, déjà en vogue à l'époque de Platon et d'Aristote, était entré dans une phase pour ainsi dire « scolastique » : repris sous la forme canonique où l'avaient fixé les grands philosophes antérieurs, il était alors devenu « lieu commun pour Plutarque, prétexte à dissertation pour Maxime de Tyr, à

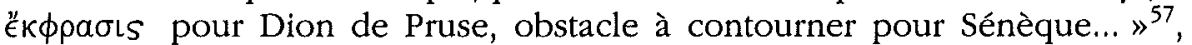
lesquels auteurs, notons-le, sont tous des écrivains plus ou moins contemporains de Luc!

On sait aussi que, selon la tradition, ce dernier était médecin. Il devait donc avoir une certaine connaissance des écrits hippocratiques. Or, à propos de la théorie de la division de l'âme en parties, le même R. Joly fait cette curieuse remarque :

Laissons l'originalité [de cette théorie] à Platon, mais avouons que cette originalité est bien mince. L'idée de diviser l'âme en deux ou trois parties était dans l'air. Galien attribue cette tripartition à Platon et aussi à Hippo-

55 Républ. II, 364b-c (trad. personnelle, faite d'après la trad. anglaise de Shorey).

57 JoLy, o.c. (n. 10), p. 171 et 189. 
crate: c'est peut-être une erreur (dans notre Corpus hippocratique en tout cas, on ne trouve rien de semblable), mais l'erreur est symptomatique ${ }^{58}$.

D'autre part, étudiant les rapports d'Hippocrate et de ses successeurs avec la philosophie de leur temps, R.O. Moon a pu écrire ceci à propos de Pythagore :

bien qu'il soit né à peu près exactement un siècle avant Hippocrate, on peut voir l'influence de sa philosophie dans plusieurs traités hippocratiques ${ }^{59}$.

Notre conclusion sera, dès lors, la suivante : même s'il est impossible de démontrer une réelle influence de la pensée de l'Antiquité classique sur Luc, ne faut-il pas convenir que ces rapprochements méritaient à tout le moins considération?

Université catholique de Louvain

Jacques Follon Institut supérieur de Philosophie 14, place du Cardinal Mercier B - 1348 LouvaIN-LA-NeUve

59 R.O. Moon, Hippocrates and His Successors in Relation to the Pbilosopby of Their Time, Londres, 1923 [réimpr. New York, 1979], p. 31-32. Incidemment, l'auteur cite explicitement (p. 42-43) l'extrait des Tusculanes de Cicéron que nous avons nous-même cité au début de cet article. 